\title{
Use of Chinese Herbal Medicine Improves Chemotherapy-Induced Thrombocytopenia among Gynecological Cancer Patients: An Observational Study
}

\author{
Yi-Hong Wu $\mathbb{D}^{1,1,2,3}$ Hsing-Yu Chen $\mathbb{D}^{1,2,4}$ Chyong-Huey Lai, ${ }^{3,5}$ \\ Chein-Shuo Yeh $\mathbb{D}^{6}{ }^{6}$ Jong-Hwei S. Pang, ${ }^{4}$ Jian-Tai Qiu, ${ }^{3,5}$ Hung-Hsueh Chou, ${ }^{3,5}$ \\ Lan-Yan Yang ${ }^{\mathbb{D}},{ }^{5,7}$ and Yu-Bin Pan $^{7}$ \\ ${ }^{1}$ Division of Chinese Internal Medicine, Center for Traditional Chinese Medicine, Chang Gung Memorial Hospital, Taoyuan, Taiwan \\ ${ }^{2}$ School of Traditional Chinese Medicine, College of Medicine, Chang Gung University, Taoyuan, Taiwan \\ ${ }^{3}$ Gynecologic Cancer Research Center, Chang Gung Memorial Hospital and Chang Gung University College of Medicine, \\ Taoyuan, Taiwan \\ ${ }^{4}$ Graduate Institute of Clinical Medical Sciences, College of Medicine, Chang Gung University, Taoyuan, Taiwan \\ ${ }^{5}$ Department of Obstetrics and Gynecology, Chang Gung Memorial Hospital and Chang Gung University College of Medicine, \\ Taoyuan, Taiwan \\ ${ }^{6}$ Graduate Institute of Traditional Chinese Medicine, Chang Gung University, Taoyuan, Taiwan \\ ${ }^{7}$ Biostatistics Unit, Clinical Trial Center, Chang Gung Memorial Hospital, Taoyuan, Taiwan \\ Correspondence should be addressed to Yi-Hong Wu; mzpjih@gmail.com
}

Received 29 March 2018; Revised 4 June 2018; Accepted 10 June 2018; Published 12 July 2018

Academic Editor: Jae Youl Cho

Copyright (c) 2018 Yi-Hong Wu et al. This is an open access article distributed under the Creative Commons Attribution License, which permits unrestricted use, distribution, and reproduction in any medium, provided the original work is properly cited.

\begin{abstract}
Background. Chemotherapy-induced thrombocytopenia (CIT) is a serious complication among patients with gynecological malignancies, yet management options are limited. This study aimed at reporting the potential of the Chang Gung platelet elevating formula (CGPEF), a prescription with a fixed proportion of Chinese herbs, for improving CIT among gynecologic cancer patients. Materials. From 1/1/2007 to 31/12/2009, a total of 23 patients with two consecutive CIT episodes $\left(\leq 100 \times 10^{3} / \mu \mathrm{L}\right)($ last cycle: C0; index cycle: $\mathrm{C} 1)$ received the CGPEF from the nadir of platelet count of $\mathrm{C} 1$ and through the subsequent chemotherapy cycles (C2 and beyond). The CGPEF was taken orally four times a day. The evolution of platelet counts of 18 patients after administration of CGPEF was analyzed ( 2 patients had different chemotherapy regimens after CGPEF, two patients discontinued CGPEF due to the flavor and the amount of CGPEF, and one patient had no further chemotherapy). Results. Most of the patients had recurrent ovarian cancer $(11 / 18,61 \%)$ with a median of 2.5 previous chemotherapy regimens, and carboplatin-based regimens were the most commonly used for these patients (13/18, 72\%). The trend of successive CIT could be reversed after taking CGPEF. Also, the platelet nadir was higher after CGPEF treatment $\left(16.5 \times 10^{3} / \mu \mathrm{L}\right.$ versus $32 \times 10^{3} / \mu \mathrm{L}$, before and after CGPEF treatment, resp., $\left.p=0.002\right)$. Moreover, the chemotherapy interval decreased from 30.5 days to 24 days. No thrombocytosis, clinical bleeding, thromboembolism, or other adverse events were found among these patients. Conclusions. The CGPEF is worthy of further large-scale, well-designed clinical trials for CIT among gynecological cancer patients.
\end{abstract}

\section{Introduction}

Thrombocytopenia is one of the most severe complications of chemotherapy, and more than one-fifth of adult patients receiving chemotherapy ever experience thrombocytopenia $[1,2]$. In addition to increased bleeding risks, CIT may force physicians to reduce chemotherapy dose, change the chemotherapy regimen, and postpone the chemotherapy schedule, although the treatment is effective [3-5]. These events may prolong hospitalization course, increase medical cost, lower quality of life, and even influence disease outcome $[3,4]$. In addition to cisplatin-based chemotherapy, regimens 
containing carboplatin, paclitaxel, and gemcitabine are regimens highly related to CIT [2]. These drugs are all commonly used for the treatment of gynecological malignancies, such as endometrial, ovarian, and cervical cancer, and therefore CIT becomes an important issue when treating gynecological cancer, especially after several cycles of chemotherapy [6].

Treatment for CIT is still a challenging work of daily clinical practice for oncologists compared to treatment for chemotherapy-induced neutropenia or anemia, which are minimized due to the application of recombinant hematopoietic factors [7]. For instance, among patients with epithelial ovarian cancer, CIT may lower the survival rate due to the 1st-line chemotherapy modification if CIT remains unmanageable [8-10]. In the past decades, several agents have been developed for relieving megakaryocyte depletion, which is the main mechanism of CIT due to direct cytotoxic or immune-mediated effects of chemotherapy drugs. Interleukin-11 (IL-11) is the only agent that is approved commercially in the United States for CIT, but the clinical use is limited due to narrow therapeutic index and significant side effects, such as edema, dyspnea, arrhythmia, syncope, and fatigue [11]. Additionally, some novel thrombopoietic stimulants have also been developed, such as recombinant human thrombopoietin (rhTPO), pegylated recombinant human megakaryocyte growth and development factor (PEG-rHuMGDF), and TPO receptor agonists $[5,12]$. The clinical use of rhTPO and PEG-rHuMGDF is limited due to the development of neutralizing antibody [5]. Eltrombopag and romiplostim are two TPO agonists ever used for CIT, but there are only a few small-scale studies about the efficacy in the recent years, and possible thrombocytosis after the medication is of concern [13-15]. Besides, the available weak evidence did not support the use of TPO agonists on CIT among patients with solid malignancies in a recent systemic review and meta-analysis [12]. For this reason, the study about alternative treatments for CIT is still needed.

Traditional Chinese medicine (TCM), one of the most commonly used complementary and alternative medicines in the world, has been used and studied increasingly yearly [16]. In Taiwan, modalities of TCM include Chinese herbal medicine (CHM), acupuncture, and massage, and all these modalities are reimbursed by the national insurance. Nearly a half of cancer patients have used TCM, and more than $90 \%$ of cancer patients have been reported to use CHM as an alternative therapy [17-19]. The effectiveness of CHM in relieving side effects due to chemotherapy, such as nausea, vomiting, and fatigue, has been reported in some reports [20-24]. Additionally, some CHM, such as Astragalus membranaceus, has been found to be beneficial for neutropenia and anemia after chemotherapy $[21,25,26]$. Nevertheless, to the best of our knowledge, reports about CHM treatments on CIT are still lacking, and the efficacy of CHM remains to be elucidated [24].

This study aims to demonstrate potential efficacy of the Chang Gung platelet elevating formula (CGPEF), a CHM formula with fixed proportion, for a series of patients with gynecological malignancies suffered from CIT. The results of this study provide the basis for further well-designed clinical trials for CGPEF on CIT patients.

\section{Methods}

2.1. Study Design. From $1 / 1 / 2007$ to $31 / 12 / 2009$, patients with gynecological malignancies who suffered from two successive episodes of CIT were referred to TCM doctor for the CGPEF treatment. The patients' eligibility is assessed by two gynecologic oncologists (Dr. Chyong-Huey Lai and Jian-Tai Qiu) in the Chang Gung Memorial Hospital (CGMH) once CIT was noted after two successive chemotherapy cycles, while CIT was defined as the platelet count lower than $100 \times 10^{3} / \mu \mathrm{L}$ after chemotherapy. The decision about eligibility was mainly based on gynecologic oncologists' clinical experiences and patients' preference. If CIT had occurred in the previous chemotherapy cycle $(\mathrm{C} 0)$ and CIT recurred at the subsequent course $(\mathrm{C} 1)$, the intervention of CGPEF would be suggested and administered from the nadir of platelet count of $\mathrm{Cl}$ and through the subsequent chemotherapy cycles (C2 and beyond) if patients agreed (Figure 1).

\subsection{The Chang Gung Platelet Elevating Formula (CGPEF) for} CIT. The CGPEF is a prescription composed of 24 kinds of CHMs from 4 classic formulas: Ren-Shen-Yang-YingTang, Gui-Pi-Tang, Gui-Lu-Er-Xian-Jiao, and Hu-Qian-Wan. These formulas have same ingredients recorded in the TCM textbooks, except tiger bone in $\mathrm{Hu}-\mathrm{Qian}-\mathrm{Wan}$, because it is strictly prohibited in Taiwan. All CHMs have been commonly used for hundreds of years and were often used by TCM doctors to treat qi, blood, and kidney deficiency, which are thought to be the main cause of CIT on TCM's viewpoint [24, 27]. The composition of the CGPEF is summarized in Table 1. All these herbs are produced by the Chuang Song-Zong Pharmaceutical Factory with good manufacturing practice during the entire observation time and are examined in detail for possible heavy metal, pesticide, or toxin. Every patient was given one pack of CGPEF, weighted 18 gm for adult weighing more than $60 \mathrm{~kg}$, four times a day. The content of CGPEF, safety issues, and possible effectiveness were well explained to these patients in detail after the eligibility was confirmed. Patients were free to choose to take the CGPEF or not or discontinue the CGPEF at any time.

2.3. Ethical Consideration. The Institutional Review Board at Chang Gung Memorial Foundation approved this study (No. 99-1241B, 102-0721C).

2.4. Outcome Assessment. The changes in platelet counts after taking the CGPEF were the primary outcome of this study, while the lab data of each patient was collected retrospectively. The timing to collect blood samples was between days 7 and 14 of the cycle of chemotherapy in $\mathrm{C} 1$ and the first day of next cycle (C2) with 3-5 days' intervals, depending on the severity of thrombocytopenia. Outcome parameters were compared between $\mathrm{C} 1$ and $\mathrm{C} 2$ (Figure 1). The secondary outcome parameters included duration of platelet counts less than $25 \times 10^{3} / \mu \mathrm{L}, 50 \times 10^{3} / \mu \mathrm{L}$, and $75 \times 10^{3} / \mu \mathrm{L}$ after chemotherapy, platelet recovery time to $50 \times 10^{3} / \mu \mathrm{L}$ and $75 \times 10^{3} / \mu \mathrm{L}$, and the interval between chemotherapies. Moreover, the need for blood transfusion was recorded as transfusion frequency and amount. 


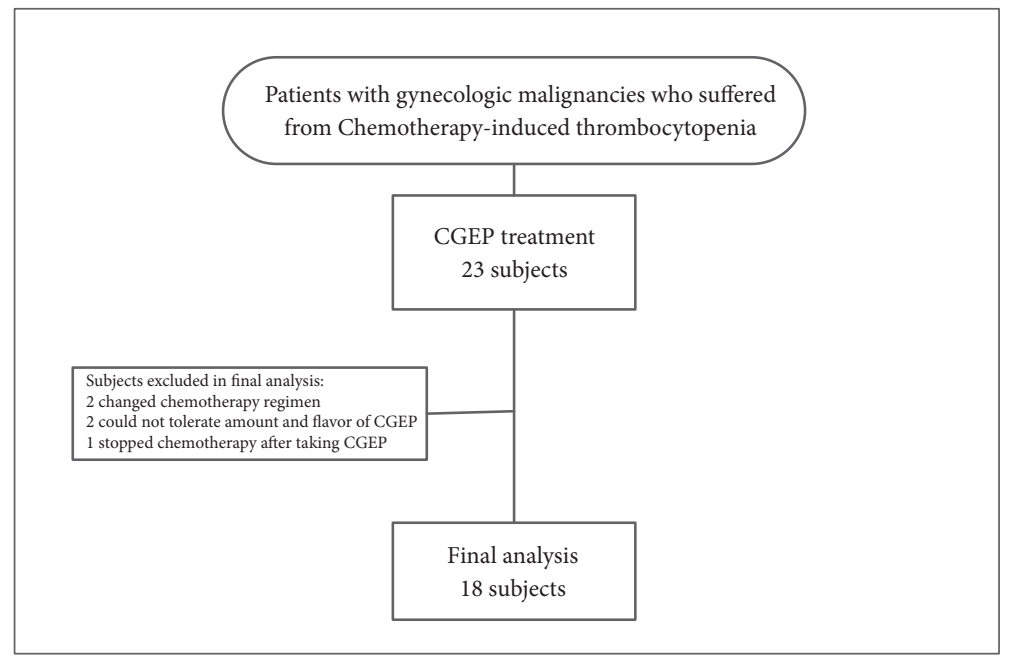

FIGURE 1: Diagram of enrollment and investigation of subjects in this study.

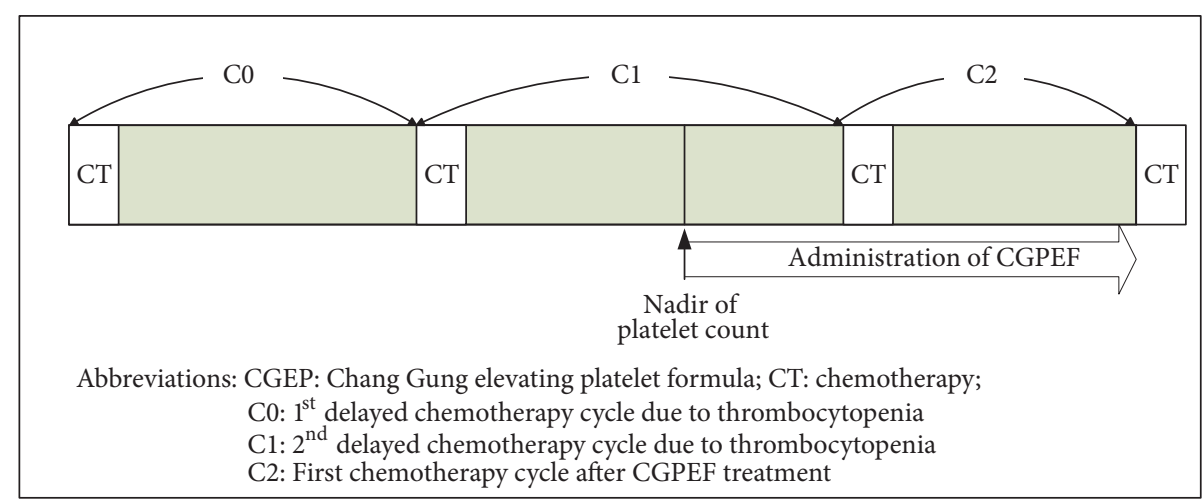

FIGURE 2: Scheme of managements and follow-up of CGPEF treatment.

2.5. Statistical Analysis. Wilcoxon signed-rank test was used to compare platelet counts, durations, and days for recovery of $\mathrm{C} 1$ and $\mathrm{C} 2$, presenting the condition of CIT before and after CGPEF treatment. All statistical calculation is done by the SPSS software and the results with $p$ value less than 0.05 were thought to be statistically significant.

\section{Results}

A total of 23 patients ever received CGPEF for their CIT, and 18 of them are included in the final analysis. Among the excluded patients, two patients could not tolerate CGPEF due to drug amount and flavor; two patients received different chemotherapy regiments when taking CGPEF and one patient did not receive further chemotherapy after CGPEF treatment (Figure 2). The characteristics of enrolled patients are listed in Table 2. These patients aged 58.06 years on average. More than $60 \%$ of patients had ovarian cancer, followed by cervical cancer $(27.78 \%)$. These patients had no other severe comorbidities, and only one patient had wellcontrolled hypertension. These patients had regionally or distantly advanced malignancies and had received at least two courses with different chemotherapy regimens for palliative intention (2.72 courses in average). Carboplatin was the most commonly used chemotherapy among these patients, about $72.2 \%$ of all patients.

The interval in chemotherapy initiation date extended to 31 days in average (median value: 30.5 days). The nadir of platelet count was about $24.1 \times 10^{3} / \mu \mathrm{L}$ (median count: $16.5 \times 10^{3} / \mu \mathrm{L}$ ) (Table 3). After CGPEF treatment, the interval of chemotherapy cycles was about nine days shorter than a previous cycle (30.5 versus 24 days in median value, $p=0.109)$. Additionally, the nadir of platelet count was also higher after treatment $\left(16.5 \times 10^{3} / \mu \mathrm{L}\right.$ versus $32.0 \times 10^{3} / \mu \mathrm{L}$ in median value, $p=0.002$ ). However, duration of nadir and recovery time did not differ significantly, although the durations were all shorter after CGPEF treatment (Table 3). Overall, the trends of thrombocytopenia after chemotherapy seemed reversible after CGPEF treatment (Figures 3 and 4), in which median value was $53 \times 10^{3} / \mu \mathrm{L} .62 .5 \%$ of patients could have nadir higher than $25 \times 10^{3} / \mu \mathrm{L}$ after chemotherapy during CGPEF treatment.

Generally, the CGPEF was well tolerated for the patients, except the intolerance to CGPEF amount and smells. Sometimes patients complained about minor abdominal fullness 
TABLE 1: Composition of Chang Gung platelet elevating formula (CGPEF).

\begin{tabular}{lc}
\hline Chinese herbal products & Weight (gm) \\
\hline Cervus nippon Temminck & 1.8 \\
Trionyx sinensis (Wiegmann) & 1.6 \\
Phellodendron chinense Schneid. & 0.7 \\
Angelica sinensis (Oliv.) Diels. & 0.7 \\
Panax ginseng C. A. Meyer & 0.7 \\
Paeonia lactiflora Pall. & 0.7 \\
Achyranthes bidentata Blume & 0.6 \\
Zingiber officinale Rosc. & 0.6 \\
Rehmannia glutinosa (Gaertn.) Libosch. & 0.5 \\
Astragalus membranaceus (Fisch.) Bunge & 0.5 \\
Atractylodes macrocephala Koidz. & 0.5 \\
Poria cocos (Schw.) Wolf & 0.5 \\
Polygala tenuifolia Willd. & 0.4 \\
Glycyrrhiza uralensis Fisch. & 0.4 \\
Citrus reticulata Blanco & 0.4 \\
Anemarrhena asphodeloides Bunge & 0.4 \\
Zizyphus jujuba Mill. & 0.3 \\
Euphoria longan (Lour.) Steud. & 0.3 \\
Zizyphus jujuba Mill. & 0.3 \\
Cinnamomum cassia Blume & 0.2 \\
Lycium barbarum L. & 0.2 \\
Schisandra chinensis (Turcz.) Baill. & 0.2 \\
Cynomorium songaricum Rupr. & 0.2 \\
Aucklandia lappa Decne. & 0.1 \\
\hline
\end{tabular}

* Each CGPEF contains 24 Chinese herbs (12.7 gm) and $5.3 \mathrm{gm}$ starch, totally $18 \mathrm{gm}$; daily dose: $72 \mathrm{gm}$.

when taking CGPEF but it recovered soon after adjusting the medication time. As for chemotherapy, hematologic disorders were the most concerned complications after chemotherapy. For leukocyte, one patient had grade IV, five patients had grade III, and seven patients had grade I-II leukopenia, without fever episodes. Additionally, for anemia, one had grade III anemia, and the other 17 patients ever experienced grade I-II anemia, according to the Common Terminology Criteria for Adverse Events v3.0.

\section{Discussion}

CGPEF may be a potentially effective alternative treatment for CIT with the significantly higher nadir of platelet count after/before CGPEF among patients with advanced gynecological malignancies. Also, CGPEF seems beneficial to improve delays in subsequent chemotherapy with marginally statistical significance. The benefit of CHM has been proposed for treating chemotherapy-related side effects; however, the evidence is still lacking, especially for CIT patients with gynecological malignancies [22]. Thrombocytopenia is one of the most important factors to postpone patient's subsequent chemotherapy since carboplatin is commonly used among these patients, as our case series [28]. Delayed chemotherapy, especially with a reduced dose, could decrease

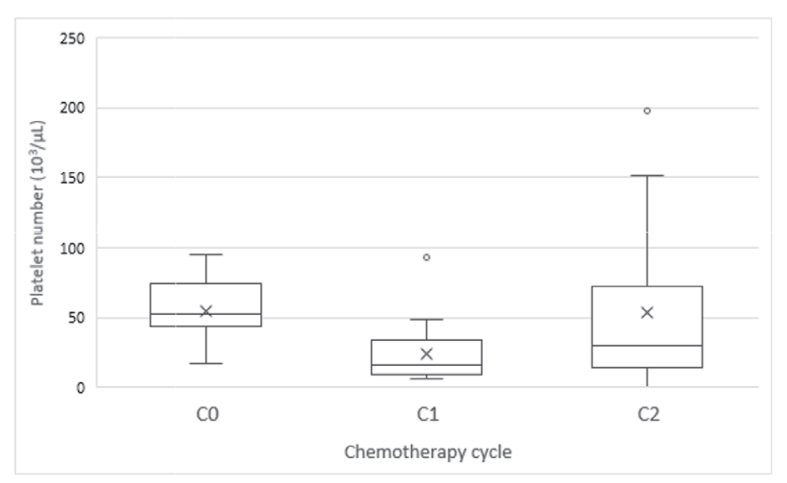

FIGURE 3: The distribution of nadir platelet count after chemotherapy (C0: the first cycle of nadir platelet count $\leq 100 \times 10^{3} / \mu \mathrm{L}$; $\mathrm{Cl}$ : the successive cycle with nadir platelet count $\leq 100 \times 10^{3} / \mu \mathrm{L} ; \mathrm{C} 2$ : the first chemotherapy course completely treated by CGPEF).

overall survival rate [8-10]. Additionally, thrombocytopenia may increase risks of gastrointestinal bleeding and even hemorrhagic stroke [1]. It is difficult in reversing the trend of CIT if chemotherapy is ongoing without any intervention in clinical practice. CGPEF appeared to reverse the trend of CIT even if chemotherapy was kept without a reduced dose and prolonged interval (Figure 3 ). The interval between chemotherapies could be shortened to near 21 days, which is the modest interval of chemotherapy, and the median platelet nadir became higher than $30 \times 10^{3} / \mu \mathrm{L},(\mathrm{C} 2)$ which is associated with much lower risks for spontaneous bleeding compared to $16.5 \times 10^{3} / \mu \mathrm{L}$ in previous chemotherapy cycle $(\mathrm{C} 1)$.

Also, to reverse the trend of CIT, the benefit of CGPEF seems comparable to current medical treatment. IL-11, the only drug for CIT which is approved by the Food and Drug Administration in the United States, can elevate nadir of platelet count from $40 \times 10^{3} / \mu \mathrm{L}$ to $60 \times 10^{3} / \mu \mathrm{L}$, while rhTPOs have been reported to elevate nadir (from $20 \times 10^{3} / \mu \mathrm{L}$ to $44 \times 10^{3} / \mu \mathrm{L}$ ) and shorten the duration of thrombocytopenia $[11,29,30]$. Moreover, through stimulating megakaryocyte, platelet transfusion can be reduced by using IL-11 without a reduction in chemotherapy dose [11, 31]. In our case series, the tendency for the duration of and transfusion rate of CIT to decrease can also be found, although not statistically significant (Table 3). The duration of platelet counts less than $50 \times 10^{3} / \mu \mathrm{L}$ and $75 \times 10^{3} / \mu \mathrm{L}$ can be shortened $2-3$ days, and it takes 1-2 days shorter to recover to a safe level of thrombocytopenia. This trend may be more prominent if CGPEF starts on the first day of the $\mathrm{C} 2$ cycle but not in the late period of the $\mathrm{Cl}$ cycle, which may improve recovery of CIT in $\mathrm{Cl}$ cycle. Nevertheless, the larger study population is still needed to stress the difference in duration and recovery rate of CIT.

Furthermore, CGPEF treatment was quite tolerable among these patients, and only two patients stopped taking CGPEF due to its flavor and amount (72gm/day). In clinical settings, CGPEF is unfavorable for CIT patients with adhesion ileus or massive ascites. For IL-11, although effective, the drug-related adverse events rate is as high as $30-50 \%$, including edema, fever, hepatitis, coagulopathy, fatigue, and arthralgia $[32,33]$. On the other hand, TPO receptor agonists 
TABLE 2: Characteristics of the patients treated with Chinese herbal medicine formula: Chang-Gung platelet elevating formula (CGPEF).

\begin{tabular}{lcc}
\hline & Count $(\%)$ & Median $(\mathrm{Q} 1 ; \mathrm{Q} 3)$ \\
\hline Age & \\
Cancer type & $59.63(49.11 ; 64.75)$ \\
Cervical cancer & $11(61.1)$ \\
Ovarian cancer & $2(11.1)$ \\
Endometrial cancer & \\
\hline Comorbidities & $1(5.6)$ \\
Hypertension & $0(0)$ \\
Diabetes mellitus & $0(0)$ \\
Ischemic heart disease & $0(0)$ \\
Cerebral vascular disease & $0(0)$ \\
Coagulopathy/bleeding disorder & $0(0)$ \\
Other malignancies & \\
\hline Preceding chemotherapy regimens & \\
Chemotherapy regimens for CGPEF treatment & $2.5(1.0 ; 4.0)$ \\
Cisplatin-based & $2(11.1)$ \\
Carboplatin-based & $13(72.2)$ \\
Taxane alone & $1(5.6)$ \\
Others & $2(11.1)$ \\
\hline
\end{tabular}

TABLE 3: Comparisons of presentations of CIT before and after using CGPEF $(n=18)$.

\begin{tabular}{|c|c|c|c|c|c|}
\hline & \multicolumn{2}{|c|}{ Before CGPEF } & \multicolumn{2}{|c|}{ After CGPEF } & \multirow{2}{*}{$p$ value } \\
\hline & Median & Q1; Q3 & Median & Q1; Q3 & \\
\hline Chemotherapy interval (days) & 30.5 & $21.75 ; 41.25$ & 24 & $15.5 ; .30 .0$ & 0.109 \\
\hline \multicolumn{6}{|l|}{ Blood transfusion } \\
\hline Times (per cycle) & 0 & $0 ; 0$ & 0 & $0 ; 0$ & 0.564 \\
\hline Amount (units per cycle) & 0 & $0 ; 0$ & 0 & $0 ; 0$ & 0.999 \\
\hline \multicolumn{6}{|l|}{ Platelet counts } \\
\hline Nadir $\left(10^{3} / \mu \mathrm{L}\right)$ & 16.5 & $8.75 ; 33.75$ & 32 & $18.5 ; 83.0$ & $0.002 *$ \\
\hline$<25 \times 10^{3} / \mu \mathrm{L}$ duration (days) & 4 & $0 ; 7.5$ & 0 & $0 ; 8$ & 0.999 \\
\hline$<50 \times 10^{3} / \mu \mathrm{L}$ duration (days) & 8.5 & $4.0 ; 13.0$ & 8 & $0 ; 14.5$ & 0.211 \\
\hline$<75 \times 10^{3} / \mu \mathrm{L}$ duration (days) & 14 & $8.5 ; 28.0$ & 10.5 & $0.5 ; 19.75$ & 0.279 \\
\hline Recover to $50 \times 10^{3} / \mu \mathrm{L}$ (days) & 9 & $5.5 ; 11.5$ & 10 & $0 ; 13.0$ & 0.232 \\
\hline Recover to $75 \times 10^{3} / \mu \mathrm{L}$ (days) & 10.5 & $6.75 ; 13.5$ & 10.5 & $0.5 ; 14.5$ & 0.629 \\
\hline Recover to $100 \times 10^{3} / \mu \mathrm{L}$ (days) & 11.5 & $9.88 ; 19.5$ & 16.25 & $10.25 ; 24.17$ & $0.050 *$ \\
\hline
\end{tabular}

* indicates $p$ value $<0.05$.

CIT: chemotherapy-induced thrombocytopenia.

with potential effects on CIT, such as eltrombopag and romiplostim, are reported to increase the risk of cerebral and portal vein thrombosis, abnormal liver function and, most seriously, myelofibrosis [34-36]. Thrombocytosis associated with the use of TPO receptor agonist became another potential drawback, since thrombocytosis may be related to poor prognosis of malignancies $[37,38]$. In the light of significantly higher serum TPO level among CIT patients [39, 40], medical treatment other than recombinant TPO and TPO receptor agonist may be needed to recover thrombocytopenia without causing possible thrombosis, myelofibrosis, and even thrombocytosis [7].
CGPEF with potential effects on CIT may be related to megakaryocyte stimulation and stem cell proliferation/differentiation [41]. The precise mechanism may be different from TPO agonist. In TCM theory, a patient with fatigue, nausea, and bone marrow suppression after chemotherapy is often diagnosed as qi and blood deficiency [27]. CGPEF was composed of 24 CHM to supplement qi and blood. Panax ginseng, one of the major ingredients of CGPEF, was usually used to supplement qi and its extract, panaxadiol saponins, was able to stimulate megakaryocytic maturation [42]. Moreover, Astragalus membranaceus and Angelica sinensis radix were reported to improve bone marrow stem cell 
(a) Changes of CT interval

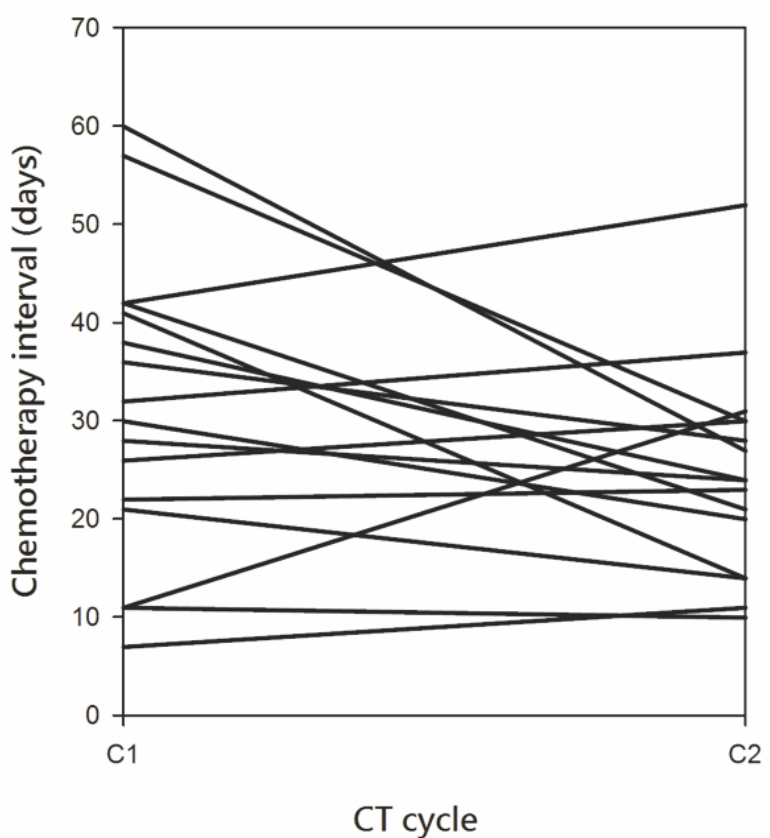

(b) Changes of nadir platelet count

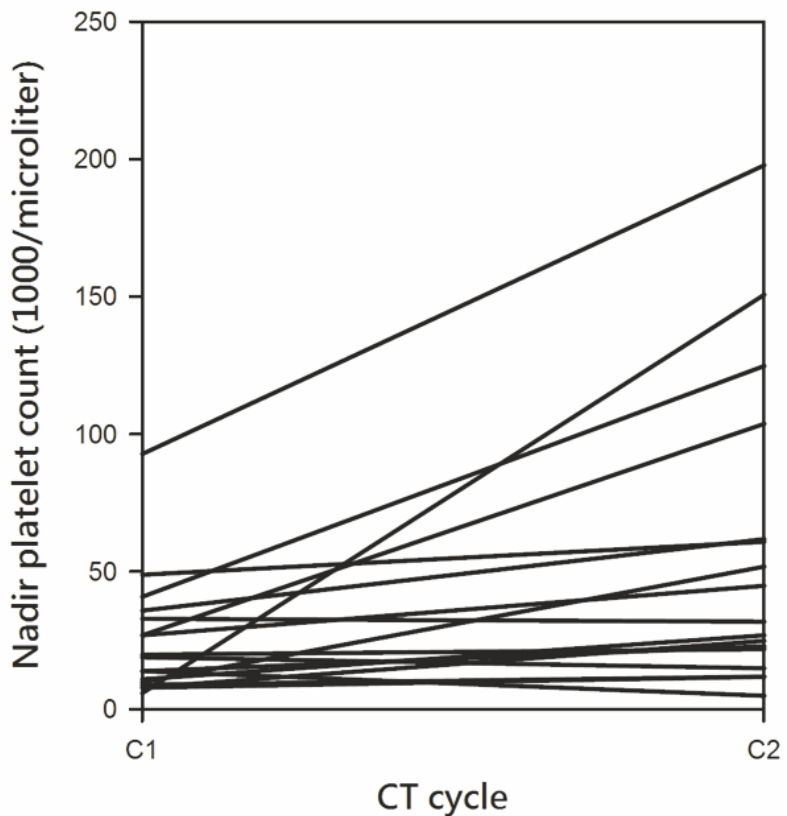

\section{Abbreviations: \\ $\mathrm{CT}$ : chemotherapy \\ C1: first delayed chemotherapy cycle due to thrombocytopenia \\ C2: subsequent chemotherapy cycle after $\mathrm{C} 1$}

FIGURE 4: The trend of changes in chemotherapy interval (A) and nadir platelet count (B) before and after CIT treated by CGPEF (C1: the second CIT after chemotherapy; C2: the first chemotherapy course completely treated by CGPEF).

proliferation [43]. In addition to improvements on thrombocytopenia, the anticancer and immunomodulation effects of Angelica sinensis [44], Panax ginseng [45-47], and Astragalus membranaceus $[48,49]$ in bench studies may imply the valuable role as adjuvant therapy in patients with gynecological malignancies and CIT.

In this observational study, we demonstrated the potential efficacy of CGPEF in treating CIT. However, there are still limitations. First, the post hoc power analysis showed that the $\beta$-error probability was about 0.23 by using Lehmann equation [50], and therefore the conclusion may be more solid if the case number could be higher. However, the important information about medication dose and duration, follow-up duration, examination scheme, and even endpoints provided in this study is crucial for further large-scale, randomized clinical trials, which are still lacking so far [12]. Second, since this is a pilot retrospective study, the differences in hemogram check-up timing may inevitably cause some errors in recording the timing and the platelet nadir count. However, because gynecologists often check hemogram earlier and more frequently once thrombocytopenia occurs, as two consecutive episodes of platelet count lower than $100 \times 10^{3} / \mu \mathrm{L}$ in $\mathrm{C} 0$ and $\mathrm{Cl}$ in this study, the error should be minimal.
Third, since this is an observation study, we cannot completely exclude the possibility that CIT recovered by nature course. However, since only patients with two successive CIT episodes were included in this study and CIT is highly associated with courses of chemotherapy [1], the spontaneous recovery of CIT seemed less possible.

\section{Conclusion}

In this study, we demonstrated that CGPEF is a potentially effective treatment for CIT. Although this study is relatively small without untreated control, we demonstrated that the median platelet nadir count after CGPEF treatment (C2) was significantly higher than before (C1). Further well-designed, double-blind, placebo-controlled clinical trial with a larger number of subjects can be done based on these preliminary results.

\section{Data Availability}

The data used to support the findings of this study are available from the corresponding author upon request. 


\section{Conflicts of Interest}

The authors declare that they have no conflicts of interest.

\section{Acknowledgments}

The authors thank Dr. Yi-Chia Wei for data collection and acknowledge the support from the Gynecology Department in the CGMH. This work was supported by grants from the Ministry of Health and Welfare, Taiwan (DOHW106-TDUB-212-113005) and grants from the Chang Gung Memorial Hospital (CMRPG3F0181).

\section{References}

[1] Y. Hashiguchi, T. Fukuda, T. Ichimura et al., "Chemotherapyinduced thrombocytopenia and clinical bleeding in patients with gynecologic malignancy," European Journal of Gynaecological Oncology, vol. 36, no. 2, pp. 168-173, 2015.

[2] M. J. T. Berg, P. M. L. A. Van Den Bemt, S. Shantakumar et al., "Thrombocytopenia in adult cancer patients receiving cytotoxic chemotherapy: results from a retrospective hospital-based cohort study," Drug Safety, vol. 34, no. 12, pp. 1151-1160, 2011.

[3] L. S. Elting, E. B. Rubenstein, C. G. Martin et al., "Incidence, cost, and outcomes of bleeding and chemotherapy dose modification among solid tumor patients with chemotherapy-induced thrombocytopenia," Journal of Clinical Oncology, vol. 19, no. 4, pp. 1137-1146, 2001.

[4] L. S. Elting, S. B. Cantor, C. G. Martin et al., "Cost of chemotherapy-induced thrombocytopenia among patients with lymphoma or solid tumors," Cancer, vol. 97, no. 6, pp. 1541-1550, 2003.

[5] S. Vadhan-Raj, "Management of chemotherapy-induced thrombocytopenia: current status of thrombopoietic agents," Seminars in Hematology, vol. 46, supplement 2, pp. S26-S32, 2009.

[6] G. L. Goldberg, D. G. Gibbon, H. O. Smith, C. DeVictoria, C. D. Runowicz, and E. R. Burns, "Clinical impact of chemotherapyinduced thrombocytopenia in patients with gynecologic cancer," Journal of Clinical Oncology, vol. 12, no. 11, pp. 2317-2320, 1994.

[7] D. J. Kuter, "Managing thrombocytopenia associated with cancer chemotherapy," Oncology, vol. 29, no. 4, pp. 282-294, 2015.

[8] J. M. Fauci, J. M. Whitworth, K. E. Schneider et al., "Prognostic significance of the relative dose intensity of chemotherapy in primary treatment of epithelial ovarian cancer," Gynecologic Oncology, vol. 122, no. 3, pp. 532-535, 2011.

[9] N. Joseph, R. M. Clark, D. S. Dizon et al., "Delay in chemotherapy administration impacts survival in elderly patients with epithelial ovarian cancer," Gynecologic Oncology, vol. 137, no. 3, pp. 401-405, 2015.

[10] S. Liutkauskiene, R. Janciauskiene, K. Jureniene, S. Grizas, R. Malonyte, and E. Juozaityte, "Retrospective analysis of the impact of platinum dose reduction and chemotherapy delays on the outcomes of stage III ovarian cancer patients," BMC Cancer, vol. 15, no. 1, article no. 105, 2015.

[11] I. Tepler, L. Elias, J. W. Smith II et al., "A randomized placebocontrolled trial of recombinant human interleukin-11 in cancer patients with severe thrombocytopenia due to chemotherapy," Blood, vol. 87, no. 9, pp. 3607-3614, 1996.

[12] X. Zhang, Y. Chuai, W. Nie, A. Wang, and G. Dai, "Thrombopoietin receptor agonists for prevention and treatment of chemotherapy-induced thrombocytopenia in patients with solid tumours," Cochrane Database of Systematic Reviews, vol. 11, 2016.

[13] A. Kellum, A. Jagiello-Gruszfeld, I. N. Bondarenko, R. Patwardhan, C. Messam, and Y. M. Kamel, "A randomized, doubleblind, placebo-controlled, dose ranging study to assess the efficacy and safety of eltrombopag in patients receiving carboplatin/paclitaxel for advanced solid tumors," Current Medical Research and Opinion, vol. 26, no. 10, pp. 2339-2346, 2010.

[14] F. Iuliano, E. Iuliano, and A. Perricelli, "Low doses of eltrombopag are safe and effective in the prophylaxis of the chemotherapy-induced thrombocytopenia (CIT)," Blood, vol. 128, no. 22, pp. 4926-4926, 2016.

[15] R. Parameswaran and G. A. Soff, "Romiplostim in a case series of chemotherapy-induced thrombocytopenia," Blood, vol. 118, no. 21, pp. 1170-1170, 2011.

[16] D. Normile, "The new face of traditional Chinese medicine," Science, vol. 299, no. 5604, pp. 188-190, 2003.

[17] L. Yi-Hsien and C. Jen-Hwey, "Use of Chinese medicine by women with breast cancer: a nationwide cross-sectional study in Taiwan," Complementary Therapies in Medicine, vol. 19, no. 3, pp. 137-143, 2011.

[18] Y.-H. Lin, K.-K. Chen, and J.-H. Chiu, "Use of Chinese medicine among prostate cancer patients in Taiwan: a retrospective longitudinal cohort study," International Journal of Urology, vol. 18, no. 5, pp. 383-386, 2011.

[19] Y.-H. Lin, K.-K. Chen, and J.-H. Chiu, "Prevalence, patterns, and costs of Chinese medicine use among prostate cancer patients: a population-based study in Taiwan," Integrative Cancer Therapies, vol. 9, no. 1, pp. 16-23, 2010.

[20] W. Taixiang, A. J. Munro, and L. Guanjian, "Chinese medical herbs for chemotherapy side effects in colorectal cancer patients," in Cochrane Database of Systematic Reviews, p. CD004540, John Wiley \& Sons, 2005.

[21] T. S. K. Mok, W. Yeo, P. J. Johnson et al., "A doubleblind placebo-controlled randomized study of Chinese herbal medicine as complementary therapy for reduction of chemotherapy-induced toxicity," Annals of Oncology, vol. 18, no. 4, pp. 768-774, 2007.

[22] M. Zhang, X. Liu, J. Li, L. He, and D. Tripathy, "Chinese medicinal herbs to treat the side-effects of chemotherapy in breast cancer patients," Cochrane Database of Systematic Reviews, no. 2, Article ID CD004921, 2007.

[23] Z. Yang, X. Liao, Y. I. Lu et al., "Add-on therapy with traditional chinese medicine improves outcomes and reduces adverse events in hepatocellular carcinoma: a meta-analysis of randomized controlled trials," Evidence-Based Complementary and Alternative Medicine, vol. 2017, 13 pages, 2017.

[24] Y. Jia, H. Du, M. Yao et al., "Chinese herbal medicine for myelosuppression induced by chemotherapy or radiotherapy: a systematic review of randomized controlled trials," Evidence-Based Complementary and Alternative Medicine, vol. 2015, 12 pages, 2015.

[25] X.-L. Zhu and B.-D. Zhu, "Mechanisms by which Astragalus membranaceus injection regulates hematopoiesis in myelosuppressed mice," Phytotherapy Research, vol. 21, no. 7, pp. 663-667, 2007.

[26] N. Yaal-Hahoshen, Y. Maimon, N. Siegelmann-Danieli et al., "A prospective, controlled study of the botanical compound mixture LCS101 for chemotherapy-induced hematological complications in breast cancer," The Oncologist, vol. 16, no. 9, pp. 1197-1202, 2011. 
[27] Y.-H. Zhou, K.-M. Wei, L.-Y. He et al., "Multi-central clinical research into treating 80 cases of chronic thrombocytopenia with qi-supplementing and yin-nourishing therapy and western medicine," Journal of Traditional Chinese Medicine, vol. 31, no. 4, pp. 277-281, 2011.

[28] A. Hitron, D. Steinke, S. Sutphin, A. Lawson, J. Talbert, and V. Adams, "Incidence and risk factors of clinically significant chemotherapy-induced thrombocytopenia in patients with solid tumors," Journal of Oncology Pharmacy Practice, vol. 17, no. 4, pp. 312-319, 2011.

[29] S. Vadhan-Raj, C. F. Verschraegen, C. Bueso-Ramos et al., "Recombinant human thrombopoietin attenuates carboplatininduced severe thrombocytopenia and the need for platelet transfusions in patients with gynecologic cancer," Annals of Internal Medicine, vol. 132, no. 5, pp. 364-368, 2000.

[30] S. Vadhan-Raj, F. Hagemeister, and L. E. Fayad, "Randomized, double-blind, placebo-controlled, dose and schedule-finding study of AMG 531 in chemotherapy-induced thrombocytopenia (CIT): results of a phase I/II study," Blood, vol. 116, no. 21, pp. 656-657, 2010.

[31] X. Du and D. A. Williams, Interleukin-11: Review of Molecular, Cell Biology, and Clinical Use, 1997.

[32] S. Wu, Y. Zhang, L. Xu et al., "Multicenter, randomized study of genetically modified recombinant human interleukin-11 to prevent chemotherapy-induced thrombocytopenia in cancer patients receiving chemotherapy," Supportive Care in Cancer, vol. 20, no. 8, pp. 1875-1884, 2012.

[33] M. S. Gordon, W. J. McCaskill-Stevens, L. A. Battiato et al., "A phase I trial of recombinant human interleukin-11 (neumega rhIL-11 growth factor) in women with breast cancer receiving chemotherapy," Blood, vol. 87, no. 9, pp. 3615-3624, 1996.

[34] C. M. Mulla, A. Rashidi, and A. B. Levitov, "Extensive cerebral venous sinus thrombosis following a dose increase in eltrombopag in a patient with idiopathic thrombocytopenic purpura," Platelets, vol. 25, no. 2, pp. 144-146, 2014.

[35] N. Kawano, S. Hasuike, H. Iwakiri et al., "Portal vein thrombosis during eltrombopag treatment for immune thrombocytopenic purpura in a patient with liver cirrhosis due to hepatitis $C$ viral infection.", Journal of clinical and experimental hematopathology : JCEH, vol. 53, no. 2, pp. 151-155, 2013.

[36] Y.-K. Kim, S.-S. Lee, S.-H. Jeong et al., "Efficacy and safety of eltrombopag in adult refractory immune thrombocytopenia," Blood Research, vol. 50, no. 1, pp. 19-25, 2015.

[37] M. De Serres, R. L. Yeager, J. E. Dillberger et al., "Pharmacokinetics and hematological effects of the PEGylated thrombopoietin peptide mimetic GW395058 in rats and monkeys after intravenous or subcutaneous administration," Stem Cells, vol. 17, no. 6, pp. 316-326, 1999.

[38] R. L. Stone, A. M. Nick, and I. A. McNeish, "Paraneoplastic thrombocytosis in ovarian cancer," The New England Journal of Medicine, vol. 366, no. 19, pp. 610-618, 2012.

[39] P. černelč, J. Kralj, U. Mlakar et al., "Serum thrombopoietin levels in acute myeloid leukaemia," Pflügers Archiv European Journal of Physiology, vol. 442, no. 7, pp. r200-r201, 2001.

[40] X.-Y. Lin, S. Hanada, K. Uozumi et al., "Serum levels of endogenous thrombopoietin and granulocyte-colony stimulating factor in patients with acute or lymphoma type adult T-cell leukemia during multicycle chemotherapy," Leukemia \& Lymphoma, vol. 43, no. 2, pp. 343-349, 2002.
[41] V. L. Udalamaththa, C. D. Jayasinghe, and P. V. Udagama, "Potential role of herbal remedies in stem cell therapy: proliferation and differentiation of human mesenchymal stromal cells," Stem Cell Research \& Therapy, vol. 7, no. 1, pp. 1-8, 2016.

[42] X. Lin, L. Yin, R. Gao et al., "The effects of panaxadiol saponins on megakaryocytic maturation and immune function in a mouse model of immune thrombocytopenia," Experimental Hematology, vol. 43, no. 5, pp. 364-373, 2015.

[43] B. Shen, L. Chen, K. Zhou, and K.-K. Jin, "Effects of astragalus and angelica on bone marrow stem cells proliferation and VEGF protein expression in vitro," Zhongguo gu shang = China journal of orthopaedics and traumatology, vol. 24, no. 8, pp. 652-655, 2011.

[44] W. Cao, X.-Q. Li, X. Wang et al., "A novel polysaccharide, isolated from Angelica sinensis (Oliv.) Diels induces the apoptosis of cervical cancer HeLa cells through an intrinsic apoptotic pathway," Phytomedicine, vol. 17, no. 8-9, pp. 598-605, 2010.

[45] H. R. Lemmon, J. Sham, L. A. Chau, and J. Madrenas, "High molecular weight polysaccharides are key immunomodulators in North American ginseng extracts: characterization of the ginseng genetic signature in primary human immune cells," Journal of Ethnopharmacology, vol. 142, no. 1, pp. 1-13, 2012.

[46] J. Li, T. Liu, L. Zhao et al., "Ginsenoside 20(S)-Rg3 inhibits the Warburg effect through STAT3 pathways in ovarian cancer cells," International Journal of Oncology, vol. 46, no. 2, pp. 775781, 2015.

[47] L.-D. Liang, T. He, T.-W. Du, Y.-G. Fan, D.-S. Chen, and Y. Wang, "GinsenosideRg5 induces apoptosis and DNA damage in human cervical cancer cells," Molecular Medicine Reports, vol. 11, no. 2, pp. 940-946, 2015.

[48] A. Nalbantsoy, T. Nesil, Ö. Yilmaz-Dilsiz, G. Aksu, S. Khan, and E. Bedir, "Evaluation of the immunomodulatory properties in mice and in vitro anti-inflammatory activity of cycloartane type saponins from Astragalus species," Journal of Ethnopharmacology, vol. 139, no. 2, pp. 574-581, 2012.

[49] G. Yin, D. Tang, J. Dai et al., "Combination efficacy of Astragalus membranaceus and Curcuma wenyujin at different stages of tumor progression in an imageable orthotopic nude mouse model of metastatic human ovarian cancer expressing red fluorescent protein," Anticancer Reseach, vol. 35, no. 6, pp. 31933207, 2015.

[50] G. Shieh, S.-L. Jan, and R. H. Randles, "Power and sample size determinations for the Wilcoxon signed-rank test," Journal of Statistical Computation and Simulation, vol. 77, no. 7-8, pp. 717724, 2007. 


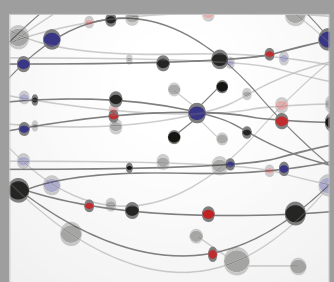

The Scientific World Journal
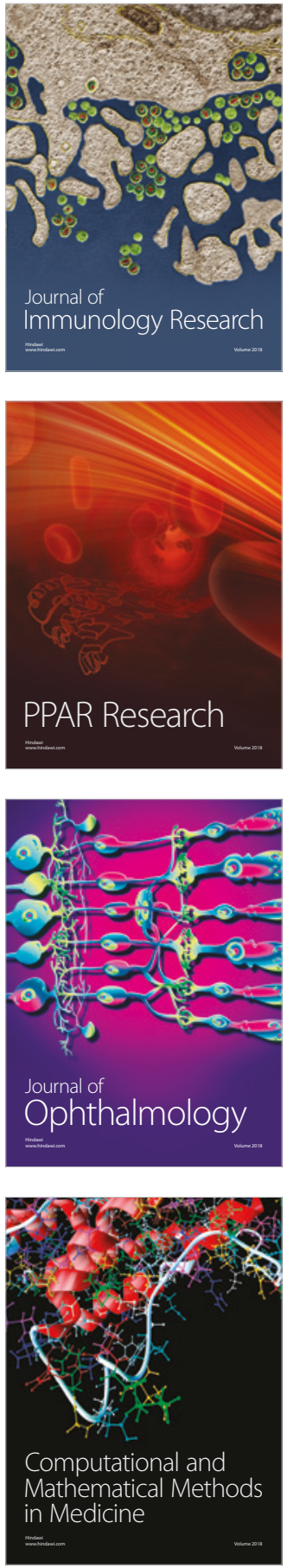

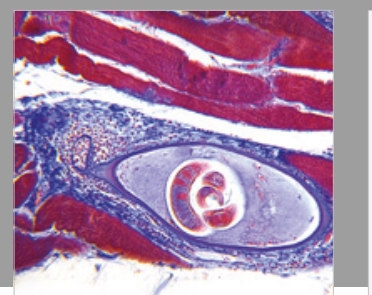

Gastroenterology Research and Practice

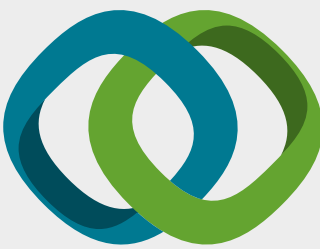

\section{Hindawi}

Submit your manuscripts at

www.hindawi.com
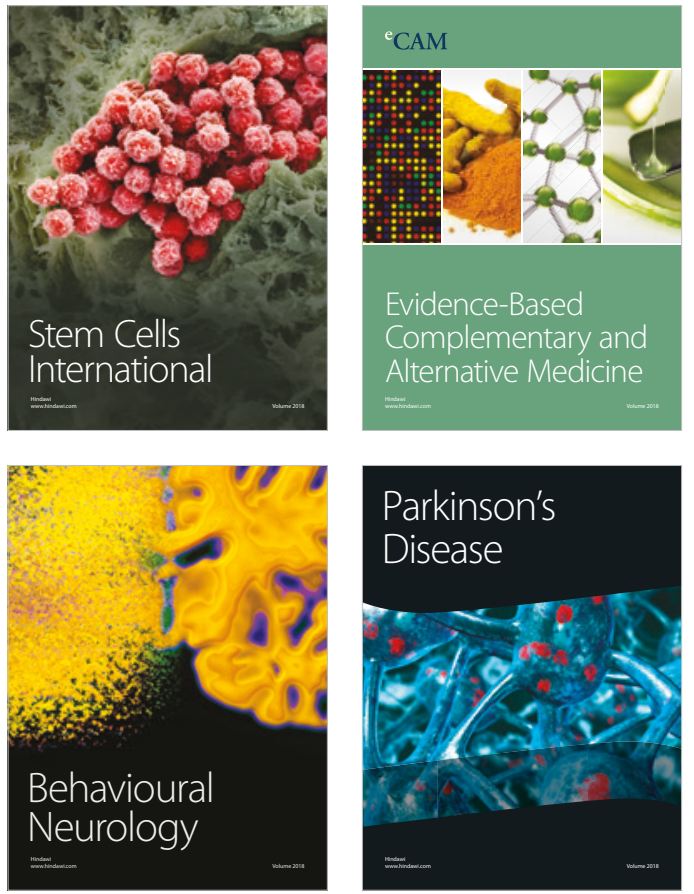

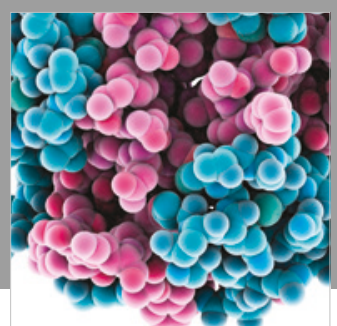

ournal of

Diabetes Research

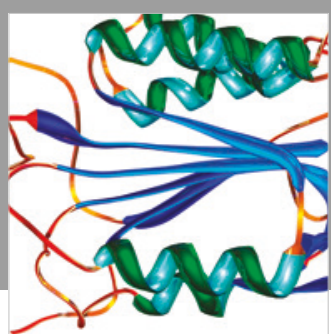

Disease Markers
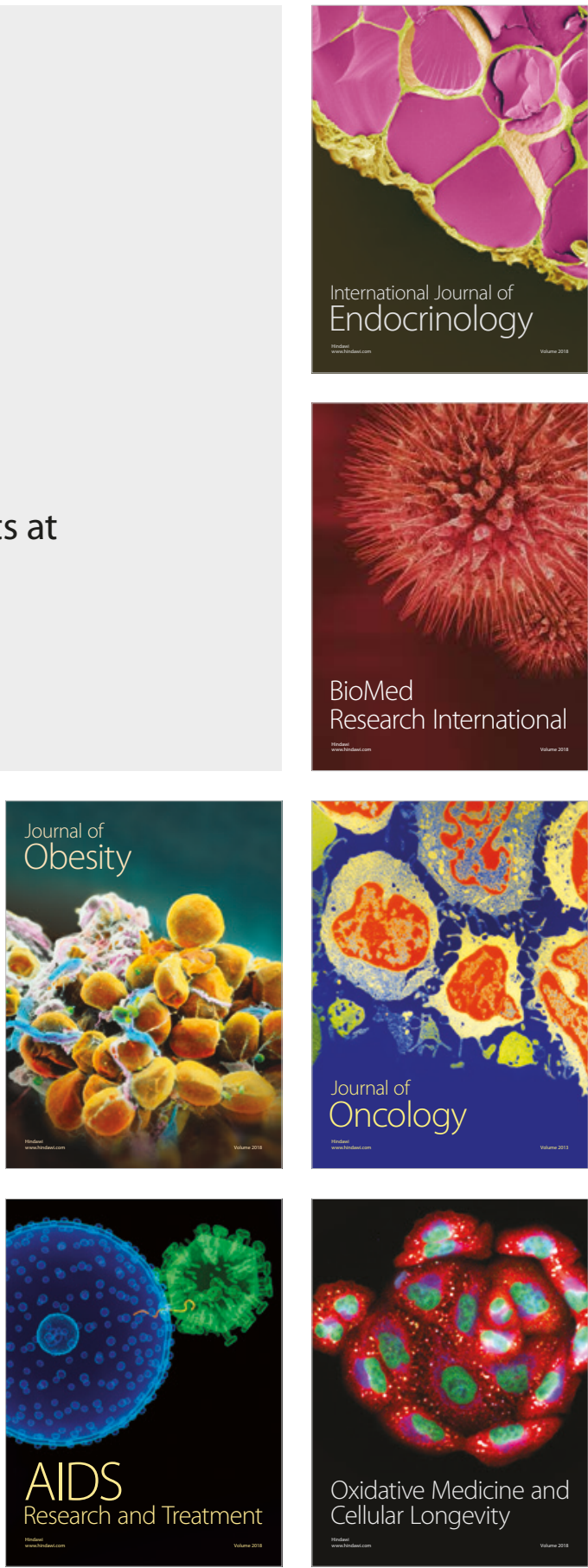